YAN-YAN QIN, Ph.D. . $^{1,2}$

E-mail: qinyanyan@cqjtu.edu.cn

HAO WANG, Ph.D. ${ }^{2}$

(Corresponding Author)

E-mail: haowang@seu.edu.cn

QUAN CHEN, Ph.D. Candidate ${ }^{2}$

E-mail: 1445212134@qq.com

BIN RAN, Ph.D. ${ }^{3}$

E-mail: bran@wisc.edu

${ }^{1}$ School of Traffic and Transportation

Chongqing Jiaotong University

Chongqing, 400074, China

2 School of Transportation

Southeast University

Nanjing, Jiangsu, 210096, China

${ }^{3}$ Department of Civil and Environment Engineering

University of Wisconsin-Madison

Madison, WI 53706, USA
Intelligent Transport Systems (ITS)

Original Scientific Paper

Submitted: 18 Apr. 2018

Accepted: 29 Aug. 2019

\title{
CAR-FOLLOWING MODEL OF CONNECTED CRUISE CONTROL VEHICLES TO MITIGATE TRAFFIC OSCILLATIONS
}

\begin{abstract}
With the aim of mitigating traffic oscillations, this paper extends a car-following model for Connected Cruise Control (CCC) systems by considering electronic throttle angles of multiple cars ahead. The linear stability condition of the proposed model is derived and numerical simulations are performed. It has been found that the proposed model is prominently better than the previous model, i.e. full velocity difference model, from the perspective of mitigating traffic oscillations. Additionally, the proposed model can also reduce fuel consumption, emissions, i.e. $\mathrm{CO}, \mathrm{HC}$ and $\mathrm{NO}_{x}$ safety risk, and improve driving comfort at the same time. Simulation results suggest that the CCC car-following control design should consider the effect of multiple electronic throttle angles from the preceding cars.
\end{abstract}

\section{KEY WORDS}

car-following model; connected cruise control; traffic oscillations; fuel consumption and emissions; safety risk; driving comfort;

\section{INTRODUCTION}

Car-following models are very essential to study dynamics of traffic flow and have been investigated for more than 60 years. During their development, many remarkable models were proposed by Pipes [1], Newell [2], Bando et al. [3], Jiang et al. [4], and Treiber et al. [5]. In addition, other improved or extended models have also been presented in previous literature $[6,7]$.

In recent years, intelligent vehicular systems, such as adaptive cruise control system [8], cooperative adaptive cruise control system [9], and connected cruise control (CCC) system [10], have been developed quickly. The investigation of their car-following models has become a hot research topic [11]. This paper focuses on the CCC car-following model. In the CCC system, a CCC car can receive information from multiple cars ahead via vehicle-to-vehicle (V2V) communication. Based on this car-following property, some models were extended by adding feedbacks into the original models [10, 12, 13]. However, it still leaves questions that need to be further solved. First, most feedbacks used in previous studies have not been validated or are difficult to be evaluated by real vehicle tests. So, their research results cannot appropriately guide the CCC car-following control design well. Moreover, previous studies usually performed simulations for car braking and starting process, as well as stability. Little research has evaluated the effect of the presented models on traffic oscillations, which is also a common traffic condition and critical to dynamics of the traffic vehicular system.

This paper presents an effort to fill the research gap by extending a CCC car-following model considering multiple cars' electronic throttle angles, which is validated to be able to be designed in CCC system tests [14-17]. Also, its linear stability analysis will be performed. Then, numerical simulations will be carried out to verify how well the proposed CCC model mitigates the traffic oscillations and what benefits will be obtained from the mitigation process, such as fuel consumption, traffic emissions, safety risk, and driving comfort. In this paper, it should be noted that traffic oscillations refer to the phenomenon that highway vehicles, instead of maintaining a steady speed, are often forced to be engaged in repeated 
deceleration - acceleration cycles [18]. Therefore, this paper mainly focuses on proposing a car-following model of CCC vehicles and evaluates the car-following model performance in terms of speeds, accelerations/ decelerations, and spacing distances under traffic oscillations. Generally speaking, the CCC car-following model belongs to the upper level of the CCC controller. Besides, the lower level of the CCC controller mainly deals with dynamic characteristics of the vehicle brake and throttle, which is beyond the scope of this paper.

\section{MODEL EQUATIONS}

Many car-following models have been developed to describe the movement of cars running on a single lane. Among them, the full velocity difference model [4] was widely utilized in literature. Here it is also employed as the surrogate model for the regular car, based on which the CCC car model will be extended. According to the previous study [19], the headway can be added to the full velocity difference model to optimize the work of model calibration. Additionally, the response time of manual drivers should be also seriously considered [20]. Hence, the car-following model of regular cars used here is written as follows:

$$
\ddot{x}_{n}\left(t+\tau_{r}\right)=\kappa\left[V\left(s_{n}(t)\right)-v_{n}(t)\right]+\frac{\lambda}{s_{n}(t)} \Delta v_{n}(t)
$$

where $x_{n}(t)$ is the position of car $n$ at time $t ; \tau_{r}$ is the driver's response time; $V($.$) is the optimal velocity func-$ tion; $v_{n}(t)$ is the speed of car $n$ at time $t ; s_{n}(t)$ is the headway between the preceding car $n-1$ and the following car $n$ at time t; while $\Delta v(t)$ is the corresponding speed difference; and $\kappa$ and $\lambda$ are sensitivity parameters.

Based on the previous studies [4, 19], the optimal velocity function is adopted as follows:

$V\left(s_{n}(t)\right)=v_{f}\left[1-\exp \left(-\frac{\alpha}{v_{f}}\left(s_{n}(t)-s_{0}\right)\right)\right]$

where $v_{f}$ is the maximum desired speed; $\alpha$ is the parameter positively related to the wave speed in a traffic jam; $s_{0}$ is the safety stopping distance.

In case of a CCC car, it receives the moving information from multiple cars ahead using V2V communication, as shown in Figure 1. The previous studies [14-17] used the information named the electronic throttle angle as the feedback for the CCC systems. Hence, this type of feedback information is also considered here. Then the extended CCC car-following model considering multiple electronic throttle angles of the preceding cars is constructed as follows:

$$
\begin{aligned}
& \ddot{x}_{n}\left(t+\tau_{c}\right)=\kappa\left[V\left(s_{n}(t)\right)-v_{n}(t)\right]+\frac{\lambda}{s_{n}(t)} \Delta v_{n}(t) \\
& +\sum_{j=1}^{m} w_{j}\left(\theta_{n-j}(t)-\theta_{n}(t)\right)
\end{aligned}
$$

where $\tau_{c}$ is the communication delay of CCC systems; $w_{j}$ is the sensitivity coefficient; $\theta_{n}(t)$ is the electronic throttle angle of car $n$ at time $t$, which is related to the speed difference and the acceleration difference [1417]:

$\theta_{n-j}(t)-\theta_{n}(t)=\frac{1}{c}\left[\ddot{x}_{n-j}(t)-\ddot{x}_{n}(t)+b\left(v_{n-j}(t)-v_{n}(t)\right)\right](4)$ where $b$ and $c$ are sensitivity coefficients whose values are 0.8 and 0.27 , respectively [17].

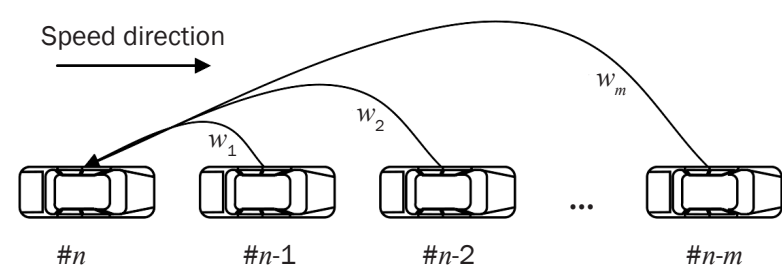

Figure 1 - CCC car receives information from $m$ cars ahead running on a single lane

\section{LINEAR STABILITY ANALYSIS}

Generally speaking, stability is usually tested for a controller and commented for the process if it is inherently stable or unstable. The car-following model of CCC vehicles belongs to the upper controller of the CCC system. Therefore, the stability of the CCC car-following model proposed in Equation 3 will be analysed in this section.

For the purpose of simplicity and more generalized derivation, Equation 3 is rewritten as follows:

$\left\{\begin{array}{l}\ddot{x}_{n}\left(t+\tau_{c}\right)=f_{n}(t)+\sum_{j=1}^{m} w_{j}\left(\theta_{n-j}(t)-\theta_{n}(t)\right) \\ f_{n}(t)=f\left(v_{n}(t), s_{n}(t), \Delta v_{n}(t)\right)\end{array}\right.$

In the initial equilibrium state, all cars run at the same speed and with headway in a homogeneous flow. Then, the presented model in Equation 5 has the position solution to the steady vehicular flow:

$\bar{x}_{n}(t)=(N-n) \bar{s}_{n}(t)+\bar{v}_{n}(t) t \quad n=1,2, \ldots, N$

where $\bar{x}_{n}(t), \bar{s}_{n}(t)$ and $\bar{v}_{n}(t)$ are position, headway, and speed, respectively, when car $n$ is running at the equilibrium state.

Supposing that a small perturbation $r_{n}(t)$ is added into the steady-state solution of car $n$ at time $t$ :

$r_{n}(t)=x_{n}(t)-\bar{x}_{n}(t)$

By conducting the first and the second derivative of both sides of Equation 7, we can obtain:

$\left\{\begin{array}{l}\dot{r}_{n}(t)=v_{n}(t)-\bar{v}_{n}(t) \\ \ddot{r}_{n}(t)=\ddot{x}_{n}(t)\end{array}\right.$ 
According to Equation 4, the angle difference $\theta_{n-i}(t)-\theta_{n}(t)$ can be calculated by substituting Equation 8 into Equation 4, as follows:

$$
\begin{aligned}
& \theta_{n-j}(t)-\theta_{n}(t)=\frac{1}{c}\left[\ddot{r}_{n-j}(t)-\ddot{r}_{n}(t)\right] \\
& +\frac{b}{c}\left[\dot{r}_{n-j}(t)-\dot{r}_{n}(t)\right]
\end{aligned}
$$

Substituting both Equations 8 and 9 into Equation 5 and linearizing the resulting equation using Taylor expansion, it follows that:

$$
\begin{aligned}
& \ddot{r}_{n}\left(t+\tau_{c}\right)=f_{n}^{v} \dot{r}_{n}(t)+f_{n}^{s}\left(r_{n-1}(t)-r_{n}(t)\right) \\
& +f_{n}^{\Delta v}\left(\dot{r}_{n-1}(t)-\dot{r}_{n}(t)\right)+ \\
& +\frac{1}{c} \sum_{j=1}^{m} w_{j}\left[\ddot{r}_{n-j}(t)-\ddot{r}_{n}(t)+b\left(\dot{r}_{n-j}(t)-\dot{r}_{n}(t)\right)\right]
\end{aligned}
$$

where $f_{n}^{v}, f_{n}^{s}$, and $f_{n}^{\Delta v}$ are partial differentials of $f_{n}(t)$ with respect to speed, headway, and speed difference at equilibrium state, respectively, which are given by

$$
\left\{\begin{array}{l}
f_{n}^{v}=\left.\frac{\partial f_{n}}{\partial v_{n}}\right|_{(\bar{v}, \bar{s}, 0)} \\
f_{n}^{s}=\left.\frac{\partial f_{n}}{\partial s_{n}}\right|_{(\bar{v}, \bar{s}, 0)} \\
f_{n}^{\Delta v}=\left.\frac{\partial f_{n}}{\partial \Delta v_{n}}\right|_{(\bar{v}, \bar{s}, 0)}
\end{array}\right.
$$

where $\bar{v}$ and $\bar{s}$ are equilibrium speed and headway, respectively.

In order to obtain the difference equation, Equation 10 is rewritten as follows:

$$
\begin{aligned}
& \dot{r}_{n}\left(t+2 \tau_{c}\right)-\dot{r}_{n}(t+\tau)= \\
& f_{n}^{v}\left[r_{n}\left(t+\tau_{c}\right)-r_{n}(t)\right]+\tau_{c} f_{n}^{s}\left[r_{n-1}(t)-r_{n}(t)\right] \\
& +f_{n}^{\Delta v}\left[r_{n-1}\left(t+\tau_{c}\right)-r_{n}\left(t+\tau_{c}\right)-r_{n-1}(t)+r_{n}(t)\right] \\
& +\frac{1}{c} \sum_{j=1}^{m} w_{j}\left[\dot{r}_{n-j}\left(t+\tau_{c}\right)-\dot{r}_{n}(t+\tau)-\dot{r}_{n-j}(t)+\dot{r}_{n}(t)\right] \\
& +b\left[r_{n-j}\left(t+\tau_{c}\right)-r_{n}\left(t+\tau_{c}\right)-r_{n-j}(t)+r_{n}(t)\right]
\end{aligned}
$$

The small perturbation $r_{n}(t)$ is rewritten as the Fourier models:

$r_{n}(t)=A \exp \left(i \alpha_{k} n+z t\right)$

where $A$ is a constant and $\alpha_{k}=\frac{2 \pi k}{N}(k=0,1, \ldots, N-1)$.

Substituting Equation 13 into Equation 12 and simplifying the resulting equation yield:

$$
\begin{aligned}
& z \exp \left(2 z \tau_{c}\right)-z \exp \left(z \tau_{c}\right)= \\
& f_{n}^{v}\left[\exp \left(z \tau_{c}\right)-1\right]+\tau_{c} f_{n}^{h}\left[\exp \left(-i \alpha_{k}\right)-1\right] \\
& +f_{n}^{\Delta v}\left[\exp \left(-i \alpha_{k}+z \tau_{c}\right)-\exp \left(z \tau_{c}\right)-\exp \left(-i \alpha_{k}\right)+1\right] \\
& +\frac{1}{c} \sum_{j=1}^{m} w_{j}\left[z \exp \left(-j i \alpha_{k}+z \tau_{c}\right)-z \exp \left(z \tau_{c}\right)-z \exp \left(-j i \alpha_{k}\right)+z\right] \\
& +\frac{b}{c} \sum_{j=1}^{m} w_{j}\left[\exp \left(-j i \alpha_{k}+z \tau_{c}\right)-\exp \left(z \tau_{c}\right)-\exp \left(-j i \alpha_{k}\right)+1\right]
\end{aligned}
$$

Let $z=z_{1}\left(i \alpha_{k}\right)+z_{z}\left(i \alpha_{k}\right)^{2}+\ldots$, then substitute it into Equation 14, we obtain the first-order and the second-order terms of coefficients in the expression of $z$, respectively, which are as follows: $z_{1}=\frac{f_{n}^{s}}{f_{n}^{v}}$

and

$$
z_{2}=\frac{f_{n}^{s}}{-\left(f_{n}^{v}\right)^{3}}\left[\frac{1}{2}\left(f_{n}^{v}\right)^{2}-f_{n}^{\Delta v} f_{n}^{v}-f_{n}^{s}+\frac{1}{2} \tau_{c} f_{n}^{s} f_{n}^{v}-\frac{b}{c} f_{n}^{v} \sum_{j=1}^{m} j w_{j}\right]
$$

The car-following model is linear stable if $z_{2}>0$; otherwise, the model is unstable. Note that $f_{n}^{v}<0$, and $f_{n}^{s}>0[21,22]$. Therefore, the linear stability condition is given by

$$
\frac{1}{2}\left(f_{n}^{v}\right)^{2}-f_{n}^{\Delta v} f_{n}^{v}-f_{n}^{s}+\frac{1}{2} \tau_{c} f_{n}^{s} f_{n}^{v}-\frac{b}{c} f_{n}^{v} \sum_{j=1}^{m} j w_{j}>0
$$

Based on Equation 11, the three partial differentials, i.e. $f_{n}^{v}, f_{n}^{s}$, and $f_{n}^{\Delta v}$, can be calculated in terms of equilibrium speed $\bar{v}$ :

$$
\left\{\begin{array}{l}
f_{n}^{v}=\kappa \\
f_{n}^{s}=\kappa \alpha\left(1-\frac{\bar{v}}{v_{f}}\right) \\
f_{n}^{\Delta v}=\frac{\lambda}{-\frac{v_{f}}{\alpha} \ln \left(1-\frac{\bar{v}}{v_{f}}\right)+s_{0}}
\end{array}\right.
$$

Additionally, when the regular human vehicular flow does not consider the response time, its linear stability condition can be obtained by simplifying Equation 17:

$\frac{1}{2}\left(f_{n}^{v}\right)^{2}-f_{n}^{\Delta v} f_{n}^{v}-f_{n}^{s}>0$

This stability condition is consistent with that in [21], which is also consistent with that in [22] if the same solving in [22] is followed:

$V^{\prime}\left(s_{n}\right)=-\frac{f_{n}^{s}}{f_{n}^{v}}$

According to Equation 17, because $f_{n}^{v}<0$ and $f_{n}^{s}>0$, the stability condition will be apt to be satisfied with the increase of both number and value of the sensitivity coefficient $w_{j}$ in the proposed CCC model. Moreover, in order to obtain a more stable CCC vehicular flow, the communication delay $\tau_{c}$ should decrease.

\section{NUMERICAL SIMULATION FOR TRAFFIC OSCILLATIONS}

At present, the implementation of real experiments on CCC platoon is not easy to conduct. Microscopic traffic simulations are usually performed to deal with this problem [11], which includes numerical simulations based on the car-following models and some microscopic traffic simulators. Since we mainly focus on the car-following model of CCC vehicles to mitigate traffic oscillations, the evaluation should be conducted using the proposed car-following model for the consistency. Therefore, this section performs numerical simulations using the car-following models of CCC vehicles and regular vehicles.

Based on the recent studies [10, 23], traffic flow oscillations can be simulated by periodical acceleration/deceleration, i.e. periodical perturbation. 
Hence, simulations in terms of periodical perturbation are performed to verify how well the proposed CCC car-following model mitigates traffic oscillations and what benefits will be obtained from this mitigation process, such as fuel consumption, traffic emissions, safety risk, and driving comfort.

According to the previous studies [19, 22], the response time of human drivers is of the order of $1.2 \mathrm{~s}$, while the communication delay of CCC systems can be of the order of $400 \mathrm{~ms}$ [24]. Additionally, the valid communication range between two cars is about $200 \mathrm{~m}$ for any driving speed at the present stage [12]. This indicates that a CCC car can receive information better from four cars ahead [9, 24]. Therefore, four sensitivity coefficients $w_{j}$ of the CCC model are considered in simulations. Other parameters of the full velocity difference model are determined by the previous calibration study [19]. Then the values of parameters used in the simulation tests are adopted as: $\tau_{r}=1.2 \mathrm{~s}, \kappa=0.629 \mathrm{~s}^{-1}$, $\lambda=4.10 \mathrm{~s}^{-1}, v_{f}=120 \mathrm{~km} / \mathrm{h}, \alpha=1.26 \mathrm{~s}^{-1}, s_{0}=2.46 \mathrm{~m}$, $\tau_{c}=0.4 \mathrm{~s}, w_{1}=0.13 \mathrm{~m} / \mathrm{s}^{-2}$ degree $^{-1}, w_{2}=0.09 \mathrm{~m} / \mathrm{s}^{-2} \mathrm{de}-$ gree $^{-1}, w_{3}=0.05 \mathrm{~m} / \mathrm{s}^{-2}$ degree ${ }^{-1}$, and $w_{4}=0.01 \mathrm{~m} / \mathrm{s}^{-2}$ degree $^{-1}$. In addition, maximum acceleration/deceleration is set as $\pm 5 \mathrm{~m} / \mathrm{s}^{2}$ in simulations and the simulation time step is $0.1 \mathrm{~s}$.

There are two cases in simulations: a platoon of ten regular cars follows a controlled leading car which performs periodical acceleration/deceleration in the one case, while ten CCC cars form the platoon in another case. The movement profile of the controlled leading car is set as follows [23]: the leading car runs at the speed of $72 \mathrm{~km} / \mathrm{h}(20 \mathrm{~m} / \mathrm{s})$ initially, which means the density is about $37.2329 \mathrm{veh} / \mathrm{km}$ at initial equilibrium state. Then it periodically changes its acceleration/ deceleration by $50 \mathrm{~s}$ with the period of $4 \mathrm{~s}$ and acceleration/deceleration $\pm 1 \mathrm{~m} / \mathrm{s}^{2}$. Finally, it recovers its original speed. The car-following performance of regular cars is conducted using the car-following model in Equation 1, while CCC car-following model in Equation 3 is used to simulate dynamics of the CCC platoon. It should be noted that the first car in the CCC platoon can only receive information from the controlled leading car. Meanwhile, the second CCC car only monitors two preceding cars, while the third CCC car monitors three cars ahead. Beginning with the fourth car in the CCC platoon, the information from four cars ahead can be received by the CCC car. This means the regular cars are simulated by using the full velocity difference model, while the CCC cars are simulated by using the new proposed car-following model. Therefore, the comparisons of simulation results between regular vehicles and CCC vehicles are actually those between the full velocity difference model and the new proposed model.

Figure 2 depicts the speed changes of cars when following the controlled leading car, in which Figure $2 a$ shows the case of regular cars, while Figure $2 b$ is the

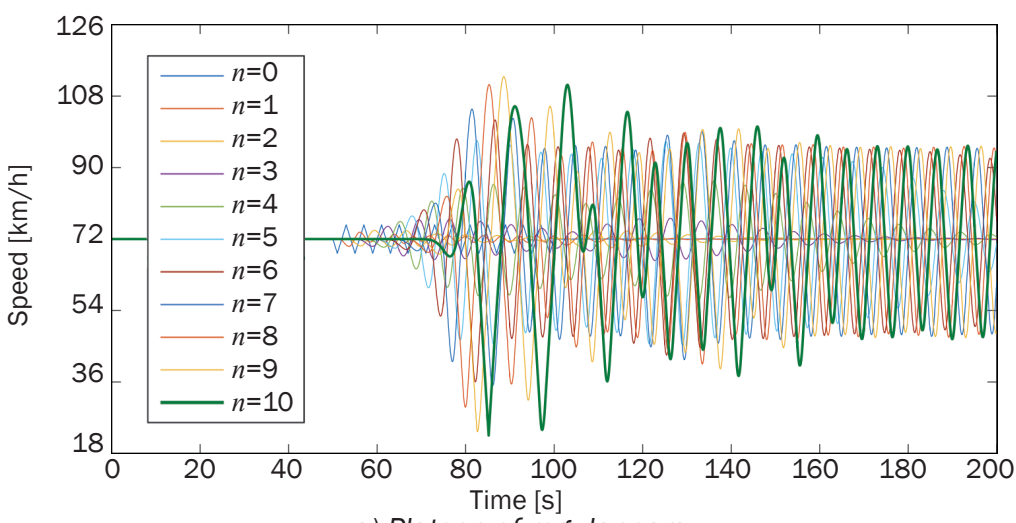

a) Platoon of regular cars

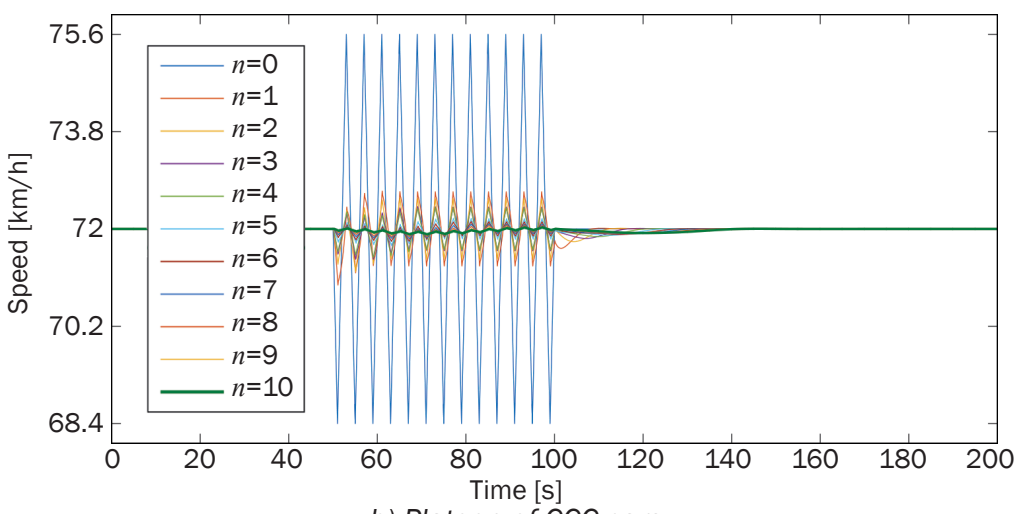

b) Platoon of CCC cars

Figure 2 - Time history of speed 


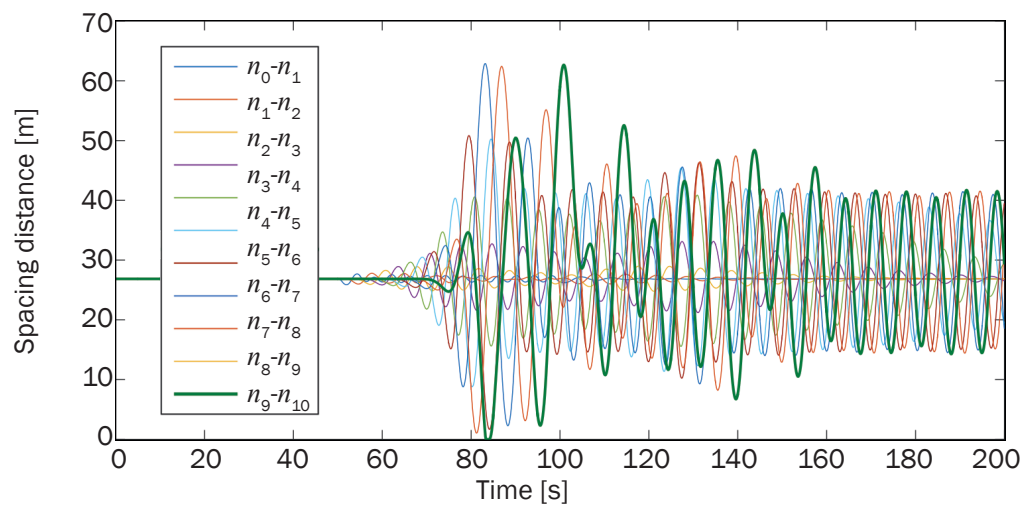

a) Platoon of regular cars

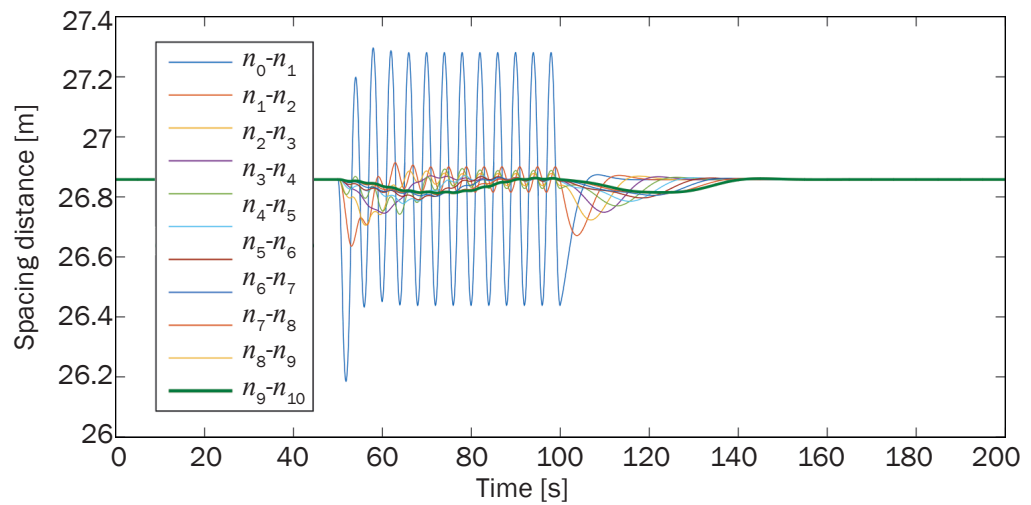

b) Platoon of CCC cars

Figure 3 - Time history of spacing distance

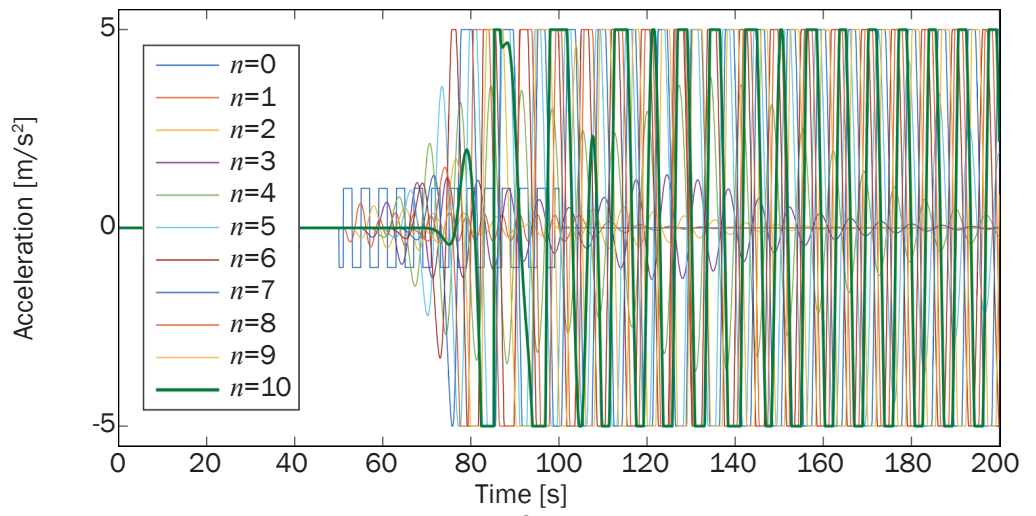

a) Platoon of regular cars

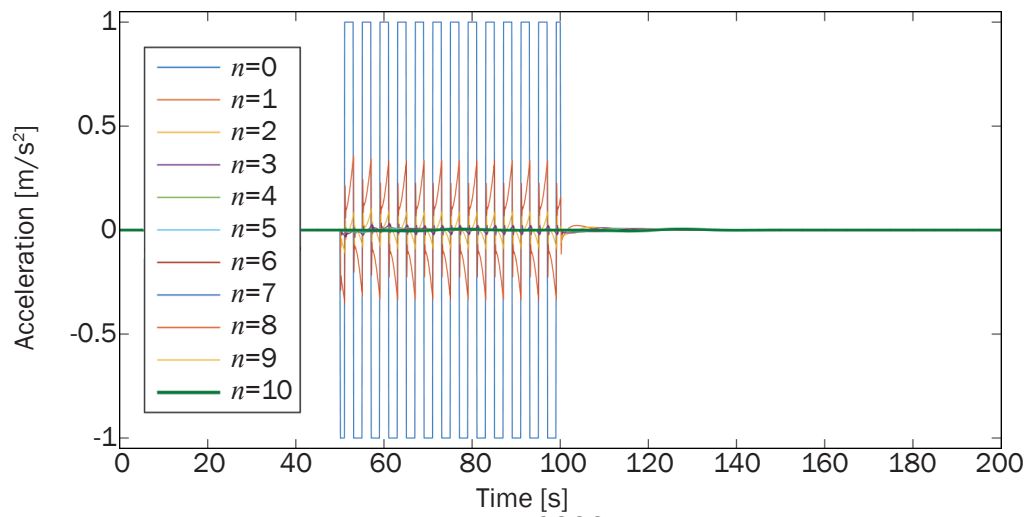

b) Platoon of CCC cars

Figure 4 - Time history of acceleration 
case of a CCC platoon. The controlled leading car is labelled by $n=0$. The following cars are labelled from $n=1$ to $n=10$. Additionally, Figures 3 and 4 show conditions of spacing distance and acceleration changes respectively, for both the regular and CCC car platoons. The label depicted in Figures 3 and 4 is the same as that in Figure 2. The simulation results in Figures 2-4 indicate that the proposed CCC car-following model can mitigate traffic oscillations well. The CCC model has stable car-following performance, while the regular cars will amplify the periodical perturbation and have difficulty for convergence. Therefore, according to the simulation results of Figures 2-4, it shows that the car-following model proposed in this paper is prominently better than the full velocity difference model from the perspective of mitigating traffic oscillations.

Traffic oscillations may result in higher fuel consumption and traffic emissions. The frequent speed and acceleration fluctuations can also cause greater safety risk and lower driving comfort. Therefore, these impacts are calculated based on the above simulations by using corresponding indicators to evaluate the benefit that the proposed CCC model contributes to mitigating traffic oscillations.

The VT-Micro model [25] was widely employed to evaluate fuel consumption and traffic emissions, such as $\mathrm{CO}, \mathrm{HC}$ and $\mathrm{NO}_{\mathrm{x}}$, under car-following models [26]. The VT-Micro model can be written as:

$$
\ln \left(M O E_{e}\right)=\sum_{i=0}^{3} \sum_{j=0}^{3} k_{i, j}^{e} v^{i}\left(\frac{d v}{d t}\right)^{j}
$$

where $M O E_{e}$ is the fuel consumption or emission rate; $k_{i, j}^{e}$ is the regression coefficient at speed power $i$ and acceleration $j ; v$ and $\frac{d v}{d t}$ are instantaneous speed and acceleration. It is pointed out that Equation 21 can explore every car fuel consumption, $\mathrm{CO}, \mathrm{HC}$ and $\mathrm{NO}_{x}$ based on different $k_{i, j}^{e}$, whose calibrated values used in $[25,26]$ are adopted here. Then, we can obtain the total fuel consumption and exhaust emissions for platoons of regular cars and CCC cars, respectively, in the progress of undergoing traffic oscillations.

In case of safety risk, the deceleration rate was employed to avoid the crash (DRAC) as the basic indicator, because it is suitable for evaluating crash risk of cars during the car-following movement [27]. DRAC was frequently utilized and is calculated as follows:

$$
D R A C_{n}^{n-1}= \begin{cases}\frac{\left(v_{n}-v_{n-1}\right)^{2}}{d_{n}^{n-1}}, & \forall v_{n}>v_{n-1} \\ 0, & \forall v_{n} \leq v_{n-1}\end{cases}
$$

where $v_{n}$ is the speed of the following car; $v_{n-1}$ is the speed of the preceding car; $d$ denotes the spacing distance between these two cars; and $D R A C_{n}^{n-1}$ stands for the deceleration rate of vehicle $n$ to avoid crashing with vehicle $n-1$. Under car-following models, car $n$ has a probability of crash with the immediately preceding car $n-1$ as well as the probability of collision with lag vehicle $n+1$. Hence, the probability of crash risk for car $n$ at time $t$, labelled by $p_{n, t}$, is calculated as follows [27]:

$$
\begin{aligned}
p_{n . t}= & p\left(D R A C_{n, t}^{n-1}>M A D R_{n, t}\right)+ \\
& p\left(D R A C_{n+1, t}^{n-1}>M A D R_{n+1, t}\right)
\end{aligned}
$$

where $p\left(D R A C_{n, t}^{n-1}>M A D R_{n, t}\right)$ is the probability of crash risk between car $n$ and car $n$-1 at time $t$, while $p\left(D R A C_{n+1, t}^{n}>M A D R_{n+1, t}\right)$ is the crash probability between car $n$ and car $n+1$. MADR denotes the maximum available deceleration rate. Then $p_{n, t}$ stands for the probability that a given $D R A C$ exceeds its $M A D R$. $M A D R$ is assumed to follow a truncated normal distribution [27], in which the mean is $8.45 \mathrm{~m} / \mathrm{s}^{2}$, the standard deviation is $1.40 \mathrm{~m} / \mathrm{s}^{2}$, the upper limit is $12.68 \mathrm{~m} / \mathrm{s}^{2}$, and the lower limit is $1.23 \mathrm{~m} / \mathrm{s}^{2}$. Based on $p_{n, t}$ in Equation 23, the average crash risk of total cars in the platoon for the whole simulation period can be calculated. It is calculated by

$R=\frac{\sum_{n=1}^{N} \sum_{t=0}^{T} p_{n, t} \Delta T}{N}$

where $R$ denotes the average safety risk, $N$ is the total number of cars in the platoon, $T$ is the total simulation time, and $\Delta T$ is the time interval which is equal to be the simulation time step.

In case of driving comfort, an indicator described by the international standard ISO 2631-1 is adopted here. It is called comfort index (CI) [28] and can be simplified under the car-following performance as follows:

$C I=\left[\frac{1}{M} \sum_{i=0}^{M}\left(a_{i}\right)^{2}\right]^{\frac{1}{2}}$

where $a_{i}$ is the $i$-th acceleration obtained by simulations for all cars and $M$ is the total number. Therefore, the driving comfort is evaluated by using the acceleration dynamics of all cars in the platoon. It is noted that a smaller value of $C I$ means more driving comfort.

Table 1 shows the evaluation results for fuel consumption, emissions, i.e. $\mathrm{CO}, \mathrm{HC}$ and $\mathrm{NO}_{x}$, safety risk, and driving comfort. It indicates that the proposed CCC model could decrease fuel consumption, $\mathrm{CO}, \mathrm{HC}$ and $\mathrm{NO}_{\mathrm{x}}$ by $54.69 \%, 47.57 \%, 70.54 \%$, and $80.94 \%$, respectively, in simulations of traffic oscillations. In addition, the CCC model can significantly reduce the safety risk and improve the driving comfort, by $96.35 \%$ and $98.43 \%$, respectively, at the same time.

We are aware that such large reductions in emissions and increases in safety may depend on the numerical simulation design of the platoon in this paper. The simulation results need to be further validated on a more complex road network and with more complex manoeuvres. In such complex environment, the reductions in emissions and increases in safety may not be so large. In fact, the car-following behaviour of vehicles 
Table 1 - Benefits of fuel consumption, emissions, safety risk, and driving comfort from the proposed CCC car-following model in traffic oscillations

\begin{tabular}{||l|c|c|c||}
\hline \multicolumn{1}{|c|}{ Types } & Platoon of regular cars & Platoon of CCC cars & Benefits \\
\hline \hline Fuel $[\mathrm{ml}]$ & 1299.2681 & 588.6947 & Saving $54.69 \%$ \\
\hline $\mathrm{CO}[\mathrm{mg}]$ & 2136.2937 & 1120.0930 & Reduced $47.57 \%$ \\
\hline $\mathrm{HC}[\mathrm{mg}]$ & 1905.3364 & 561.3823 & Reduced $70.54 \%$ \\
\hline $\mathrm{NO}_{\mathrm{x}}[\mathrm{mg}]$ & 4645.7219 & 885.5000 & Reduced $80.94 \%$ \\
\hline Safety risk & 1.8707 & 0.0683 & Reduced $96.35 \%$ \\
\hline Driving comfort $\left[\mathrm{m} / \mathrm{s}^{2}\right]$ & 2.9318 & 0.0460 & Improved $98.43 \%$ \\
\hline
\end{tabular}

can be considered as the base unit of complex road network and manoeuvres. Therefore, the qualitative conclusion that the proposed model of CCC system has positive impact on the traffic flow dynamics should be the same. Moreover, real experiments are a more reliable evaluation method. They are not available at present and should be seriously considered in the future.

\section{CONCLUSION}

This paper proposes a car-following model for CCC cars taking into consideration the electronic throttle angles of multiple cars ahead. The linear stability condition is derived. The simulation results indicate that the proposed CCC car-following model can mitigate traffic oscillations well. In addition, the proposed CCC model can reduce fuel consumption and emissions, i.e. $\mathrm{CO}, \mathrm{HC}$ and $\mathrm{NO}_{x}$ by more than about $50 \%$, respectively, when the platoon of ten CCC cars is compared with that of regular cars in the progress of undergoing traffic oscillations. At the same time, the CCC platoon can significantly reduce the safety risk and improve the driving comfort by more than $90 \%$, respectively.

However, the numerical simulations conducted in this paper have not been testified by empirical data. In view of the limitations, empirical traffic oscillations data should be used to be the movement profile of the controlled leading car in the next step, thereby deeply studying the effect of the proposed model on the traffic oscillations.

\section{ACKNOWLEDGEMENTS}

This work was supported by the National Natural Science Foundation of China (51478113), the National Key R\&D Program in China (Grant No.2016YFB0100906), the Science and Technology Research Program of Chongqing Municipal Education Commission (KJQN201900730), and the Research Foundation for Talented Scholars of Chongqing Jiaotong University (19JDKJC-C003).
秦严严, 王昊, 陈全, 由斌

平滑交通震荡的网联巡航控制车辆跟驰模型

\section{摘要}

考虑多前车电子阀门角度信息, 构建网联巡航控制系 统跟驰模型, 以平滑交通震荡。针对所提模型推导线性稳 定性条件, 同时进行数值仿真实验。研究结果表明, 所 提模型比原模型（全速度差模型）在平滑交通震荡方面 性能更优。并且, 所提模型可以降低燃油消耗与 $\mathrm{CO} 、 \mathrm{H}$ C、NOX的排放, 以及安全风险, 同时又可提升驾驶舒适 性。研究结果建议网联巡航控制系统跟驰控制设计可考虑 多前车电子阀门角度信息的反馈作用。

\section{关键词}

跟驰模型; 网联巡航控制; 交通震荡; 油耗与排放; 安全 风险; 驾驶舒适性；

\section{REFERENCES}

[1] Pipes LA. An operational analysis of traffic dynamics. Journal of Applied Physics. 1953;24(3): 274-281.

[2] Newell GF. Nonlinear effects in the dynamics of car following. Operations Research. 1961;9(2): 209-229.

[3] Bando M, Hasebe K, Nakayama A, et al. Dynamical model of traffic congestion and numerical simulation. Physical Review E. 1995;51(2): 1035-1042.

[4] Jiang R, Wu Q, Zhu Z. Full velocity difference model for a car-following theory. Physical Review E. 2001;64(1): 017101.

[5] Treiber M, Hennecke A, Helbing D. Congested traffic states in empirical observations and microscopic simulations. Physical Review E. 2000;62(2): 1805-1824.

[6] Wang H, Wang W, Chen J. General Newell model and related second-order expressions. Transportation Research Record: Journal of the Transportation Research Board. 2011;2260: 42-49.

[7] Wang H, Li Y, Wang W, et al. Optimal velocity model with dual boundary optimal velocity function. Transportmetrica B: Transport Dynamics. 2017;5(2): 215-232.

[8] Ntousakis IA, Nikolos IK, Papageorgiou M. On microscopic modelling of adaptive cruise control systems. Transportation Research Procedia. 2015; 6: 111-127.

[9] Milanés V, Shladover SE. Modeling cooperative and 
autonomous adaptive cruise control dynamic responses using experimental data. Transportation Research Part C: Emerging Technologies. 2014;48: 285-300.

[10] Qin YY, Wang H. Analytical framework of string stability of connected and autonomous platoons with electronic throttle angle feedback. Transportmetrica A: Transport Science. 2018; 1-22. Available from: doi:10.1080 /23249935.2018.1518964

[11] Mahmassani HS. 50th anniversary invited article-autonomous vehicles and connected vehicle systems: Flow and operations considerations. Transportation Science. 2016;50(4): 1140-1162.

[12] Ge Jl, Orosz G. Dynamics of connected vehicle systems with delayed acceleration feedback. Transportation Research Part C: Emerging Technologies. 2014;46: 46-64.

[13] Tang TQ, Li CY, Huang HJ. A new car-following model with the consideration of the driver's forecast effect. Physics Letters A. 2010;374(38): 3951-3956.

[14] Ioannou P, Xu Z. Throttle and brake control systems for automatic vehicle following. Journal of Intelligent Transportation Systems. 1994;1(4): 345-377.

[15] Li K, Ioannou P. Modeling of traffic flow of automated vehicles. IEEE Transactions on Intelligent Transportation Systems. 2004;5(2): 99-113.

[16] Fernandes P, Nunes U. Multiplatooning leaders positioning and cooperative behavior algorithms of communicant automated vehicles for high traffic capacity. IEEE Transactions on Intelligent Transportation Systems. 2015;16(3): 1172-1187.

[17] Li Y, Zhang L, Peeta S, et al. A car-following model considering the effect of electronic throttle opening angle under connected environment. Nonlinear Dynamics. 2016;85(4): 2115-2125.

[18] Li XP, Cui JX, An S, et al. Stop-and-go traffic analysis: theoretical properties, environmental impacts and oscillation mitigation. Transportation Research Part B: Methodological. 2014;70: 319-339.

[19] Wang H, Wang W, Chen J, et al. Estimating equilibrium speed-spacing relationship from dynamic trajectory data. Proceedings of the $91^{\text {rd }}$ Annual Meeting of the Transportation Research Board. Washington DC: TRB; 2012.

[20] Treiber M, Kesting A, Helbing D. Influence of reaction times and anticipation on stability of vehicular traffic flow. Transportation Research Record: Journal of the Transportation Research Board. 2007;1999: 23-29.

[21] Wilson RE. Mechanisms for spatio-temporal pattern formation in highway traffic models. Philosophical Transactions of the Royal Society of London A: Mathematical, Physical and Engineering Sciences. 2008;366(1872): 2017-2032.

[22] Treiber M, Kesting A. Traffic Flow Dynamics: Data, Models and Simulation. Berlin, Heidelberg: Springer-Verlag; 2013.

[23] Gong S, Shen J, Du L. Constrained optimization and distributed computation based car following control of a connected and autonomous vehicle platoon. Transportation Research Part B: Methodological. 2016;94: 314-334

[24] Shladover SE, Nowakowski C, Lu XY, et al. Cooperative adaptive cruise control: Definitions and operating concepts. Transportation Research Record: Journal of the Transportation Research Board. 2015;2489: 145-152.

[25] Ahn K, Rakha H, Trani A, et al. Estimating vehicle fuel consumption and emissions based on instantaneous speed and acceleration levels. Journal of Transportation Engineering. 2002;128(2): 182-190.

[26] Tang TQ, Huang HJ, Shang HY. Influences of the driver's bounded rationality on micro driving behavior, fuel consumption and emissions. Transportation Research Part D: Transport and Environment. 2015;41: 423-432.

[27] Meng Q, Weng J. Evaluation of rear-end crash risk at work zone using work zone traffic data. Accident Analysis \& Prevention. 2011;43(4): 1291-1300.

[28] Paddan GS, Griffin MJ. Evaluation of whole-body vibration in vehicles. Journal of Sound and Vibration. 2002;253(1): 195-213. 